\title{
Antimicrobial resistance pattern of Pseudomonas aeruginosa in a tertiary level hospital in Southern India
}

\author{
Dinesh Kaliyamoorthi ${ }^{1}$, K. Sharanya ${ }^{2, *}$, Chitralekha Saikumar ${ }^{3}$, G. Sridhar ${ }^{4}$, Boopathy ${ }^{5}$ \\ ${ }^{1}$ Associate Professor, ${ }^{2}$ Assistant Professor, ${ }^{3,4}$ Professor, ${ }^{5}$ Statistician, Dept. of Microbiology, ${ }^{1-3}$ Sree Balaji Medical College and \\ Hospital, Chennai, Tamil Nadu, ${ }^{4,5}$ Shri Sathya Sai Medical College and Research Institute, Nellikuppam, Tamil Nadu, India
}

*Corresponding Author:

Email: sharuisalways@yahoo.com

\begin{abstract}
Introduction: Pseudomonas have now a days become a major cause of nosocomial and community acquired infections. They are widely distributed in the hospital environment which makes them difficult to eradicate. P.aeruginosa has become the leading cause of hospital acquired infection because of its recent trend to gain resistance to most of the drugs used against it causing a multidrug resistance. Therefore it is necessary to know the changing pattern so as to achieve a good therapy for pseudomonas infection. Therefore this study was conducted to determine the antimicrobial resistance pattern of P.aeruginosa with different parameters from the patients attending the tertiary level hospital in Chennai.

Objective: The aim of the study was to enumerate the antimicrobial sensitivity pattern of Pseudomonas infection from various samples in a tertiary care hospital.

Materials and Methods: The study was carried out in Microbiology department of a tertiary care hospital during the period of February 2016 to January 2017. A total of 93 non repetitive clinical isolates of P.aeruginosa were collected from different samples.

Result: In the present study the males 51(54.8\%) were more affected than females 42(45.2\%).Age distribution showed more to be affected among adults of age 40 and above. Amikacin, Ciprofloxacin and Gentamicin shows $96.8 \%$ sensitivity. Among Cephalosporins highest resistance was observed among $1^{\text {st }}$ generation cefazolin $(83.9 \%)$ followed by $2^{\text {nd }}$ generation Cefotaxime $(17.2 \%)$ and Ceftazidime $(23.7 \%)$ the least was seen among $4^{\text {th }}$ generation Cefepime which showed $6.5 \%$ resistance.All isolates were sensitive to Imipenem.

Conclusion: Restriction of antibiotic usage must be formatted by each institution, to combat the emergence of multi drug resistant $P$. aeruginosa. Imipenem was the only anti-pseudomonal drug against which all isolates of $P$. aeruginosa were fully sensitive. We recommend a restricted and a more rational use of this drug in this hospital setting
\end{abstract}

Keywords: P.aeruginosa, Antimicrobial resistance, Hospital acquired infection.

\section{Introduction}

Infection by Pseudomonas has now a days become a major threat among the nosocomial and community acquired infectionsas they are widely distributed in the hospital environment and are difficult to eradicate. ${ }^{1}$ The clinically important species of pseudomonas are P.aeuroginosa, P.putida, P. fluroscence, P.stutzeri, P. alcaligenes, P.pseudoalcaligenes, P.putrefaciens, and P.mendocina.. However the most important among these is P.aeruginosa, as the rate of infection produced by other species are far less when compared to the infections produced by the P.aeruginosa.

The resistance produced by Pseudomonas is complex and has varied mechanisms. P.aeruginosa is notorious as it is resistant to many structurally unrelated antimicrobial drugs, this property may be attributed to low permeability of its outer membrane, acquired resistance through gene from other organism via the plasmids, transposons, bacteriophages, and biofilm production. ${ }^{2,3}$ Despite the advances in medical and surgical care and availability of wide range of antipseudomonal agents, treating the infections have become a challenge. Infections produced by ESBL(Extended spectrum beta lactamases), MDR (Multi drug resistance), MBL(Metallo beta lactamases) producing P.aeruginosa strains is rising in an alarming rate.This creates a serious health problem resulting in the increased burden of mortality, morbidity, and high health care cost.

The resistance pattern shown by pseudomonas is not the same in all countries. The highest is seen Turkey(7093\%), followed by India (43-68\%), Bangladesh(40$86 \%)$, Pakistan $(35-40 \%)$, Iran $(36-75 \%)$ but low in Singapore(10-23\%), West Indies(2.6-12.3\%) and Malaysia (6.7-23\%). There is also a trend in which the resistance percentage is increasing in some countries as seen in United Kingdom where the resistance percentage was $8 \%$ in 2001 and has raised to $43 \%$ in 2003, in Saudi Arabia it has increased from 10\% 1998 to $40 \%$ in 2004.

P.aeruginosa has become the major cause of hospital acquired infection because of its recent trend to gain resistance to most of the drugs used against it causing a multidrug resistance. Therefore it is necessary to know the changing pattern so as to achieve a good therapy of pseudomonas infection. Therefore this study was conducted to determine the antimicrobial resistance pattern of P.aeruginosa with different parameters from the patients attending the tertiary level hospital in Chennai. 


\section{Materials and Methods}

The study was carried out in a tertiary care hospital during the period of February 2016 to January 2017. A total of 93 non repetitive clinical isolates of P.aeruginosa were obtained from various specimens like high vaginal swab, sputum, CSF, body fluids, pus, ear swab and eye swab of patients attending the out patient departments \& in-patients from various wards including Intensive care units. Ethical committee clearance was obtained from the Institute and informed consent was obtained from all the patients. It is a prospective study and data was analysed statistically using SPSS version 23 .All the samples were inoculated onto nutrient agar plate, blood agar plate, Maconkey agar plate and incubated at $37^{\circ} \mathrm{C}$ overnight. The colonies were tested for oxidase test and other biochemical tests like Indole test, Citrate test, Urease test, Triple Sugar iron agar test and Mannitol motility media test were done for identification.

The antibiotic sensitivity test was performed by Kirby Bauer disc diffusion technique with commercially available discs (Hi-Media) on Muller Hinton Agar using Gentamycin (10mcg), Amikacin (30mcg), Ciprofloxacin (5mcg),Cefazolin, Ceftazidime (30mcg), Cefotaxime (30 mcg), Imipenem (10mcg), Cefepime (30 mcg).
Results were interpreted according to the Clinical and Laboratory Standards Institute (CLSI) guidelines.

\section{Results}

In this study among various clinical samples a total of 93 isolates of P.aeruginosa were identified.

Table 1: Distribution of age among the clinical isolates of P.aeruginosa

\begin{tabular}{|l|c|c|}
\hline \multicolumn{1}{|c|}{ Age } & Frequency & Percentage \\
\hline $0-5$ & 2 & 2.2 \\
\hline $5-20$ & 7 & 7.5 \\
\hline $20-40$ & 27 & 29.0 \\
\hline Above 40 & 57 & 61.3 \\
\hline Total & 93 & 100.0 \\
\hline
\end{tabular}

Fig. 1:Sex distribution among clinical isolates of P.aeruginosa

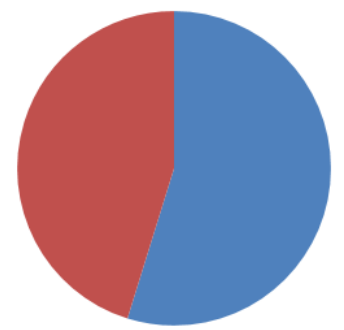

MALE FEMALE

Table 2: Distribution of sample among clinical isolates of P.aeruginosa

\begin{tabular}{|l|c|c|}
\hline \multicolumn{1}{|c|}{ Sample } & Frequency & Percentage \\
\hline Sputum & 46 & 49.5 \\
\hline HighVaginal Swab & 14 & 15.1 \\
\hline Pus & 21 & 22.6 \\
\hline CSF & 1 & 1.1 \\
\hline Ear swab & 5 & 5.4 \\
\hline Eye swab & 3 & 3.2 \\
\hline Suction tube & 1 & 1.1 \\
\hline Drainage Tube & 1 & 1.1 \\
\hline Throat Swab & 1 & 1.1 \\
\hline Total & 93 & 100.0 \\
\hline
\end{tabular}

Fig. 2: Antibiotic Susceptibility patterns of P.aeruginosa clinical isolates nesistance $\quad$ Sensitivity

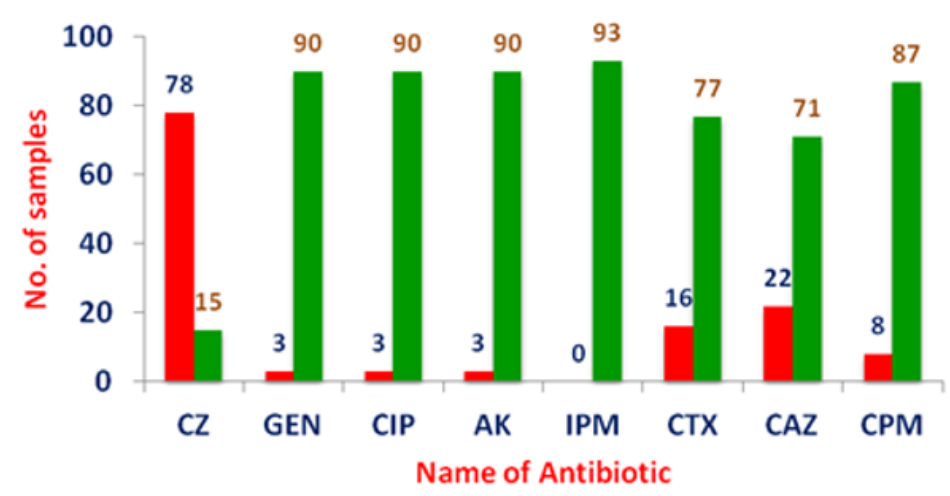


Table 3: Age Vs Sample using chi-square test

\begin{tabular}{|l|c|c|c|c|c|c|c|c|c|c|}
\hline \multirow{2}{*}{ Age } & \multicolumn{9}{|c|}{ Sample } & Ear \\
\cline { 2 - 12 } & Sputum & $\begin{array}{c}\text { High Vaginal } \\
\text { Swab }\end{array}$ & Pus & CSF & $\begin{array}{c}\text { Eye } \\
\text { swab } \\
\text { swab }\end{array}$ & $\begin{array}{c}\text { Suction } \\
\text { tube }\end{array}$ & $\begin{array}{c}\text { Drainage } \\
\text { Tube }\end{array}$ & $\begin{array}{c}\text { Throat } \\
\text { swab }\end{array}$ & Total \\
\hline$<5$ & 0 & 0 & 1 & 0 & 0 & 1 & 0 & 0 & 0 & 2 \\
\hline $05-20$ & 1 & 1 & 0 & 0 & 4 & 0 & 0 & 0 & 1 & 7 \\
\hline $20-40$ & 9 & 7 & 9 & 0 & 1 & 0 & 1 & 0 & 0 & 27 \\
\hline$>40$ & 36 & 6 & 11 & 1 & 0 & 2 & 0 & 1 & 0 & 57 \\
\hline Total & 46 & 14 & 21 & 1 & 5 & 3 & 1 & 1 & 1 & 93 \\
\hline
\end{tabular}

Fisher Exact test value is: 56.536

$P$ value: .000 (It is statistically significant)

Table 4: Sex Vs Sample using chi-square test

\begin{tabular}{|l|c|c|c|c|c|c|c|c|c|c|}
\hline Sex & \multicolumn{9}{|c|}{ Sample } & Total \\
\cline { 2 - 11 } & Sputum & $\begin{array}{c}\text { High } \\
\text { Vaginal } \\
\text { Swab }\end{array}$ & Pus & CSF & $\begin{array}{c}\text { Ear } \\
\text { swab }\end{array}$ & $\begin{array}{c}\text { Eye } \\
\text { swab }\end{array}$ & $\begin{array}{c}\text { Suction } \\
\text { tube }\end{array}$ & $\begin{array}{c}\text { Drainage } \\
\text { Tube }\end{array}$ & $\begin{array}{c}\text { Throat } \\
\text { swab }\end{array}$ & \\
\hline Male & 26 & 0 & 16 & 0 & 4 & 3 & 1 & 1 & 0 & 51 \\
\hline Female & 20 & 14 & 5 & 1 & 1 & 0 & 0 & 0 & 1 & 42 \\
\hline Total & 46 & 14 & 21 & 1 & 5 & 3 & 1 & 1 & 1 & 93 \\
\hline
\end{tabular}

Fisher Exact test value is: 30.077

$\mathrm{P}$ value: .000 (It is statistically significant)

\section{Discussion}

Pseudomonas aeruginosa has emerged as one of the significant causes of morbidity and mortality among in-patients. In this study, a total of 93 isolates of P.aeruginosawas isolated and identified from various clinical samples, from the hospitalized patients and their antimicrobial susceptibility patterns were determined. Most of them belonged to older age group of above $40(61.3 \%)$ and found to be least $2(2.2 \%)$ among children $0-5$ years. A similar finding was observed in another study which showed $1.78 \%$ of pseudomonas infection among children which increased with age of 40 and above. ${ }^{4}$

A study conducted in Chennai showed the age distribution of pseudomonas infection among diabetics to be ranging between 36-75 years which is comparable to our study. ${ }^{5}$ Another study by Golshani $\mathrm{Z}$ et al in the year 2012 in Iran showed the mean age of all the patients infected with Pseudomonas aeruginosa to be 45 years. ${ }^{6}$ Another recent study conducted in 2016 also shows majority of patients with P.aeruginosa infection were above 60 years of age. ${ }^{7}$

In the present study the males 51(54.8\%) were more affected than females 42(45.2\%). A similar pattern was seen in the stuy by Golshani $\mathrm{Z}$ et al which showed 56\% males and $44 \%$ to be females. ${ }^{6}$ Another study among diabetic patients also shows concordance to our study with Male:Female ratio of $2: 1 .^{5}$ A recent study also reveals that males were infected more than females with P.aeruginosa (59.3\%). ${ }^{7}$

Regarding Clinical specimens majority of P.aeruginosa strains were isolated from sputum specimen $(49.5 \%)$ followed by pus specimen $(22.6 \%)$ and high vaginal swab(15.1\%).A similar finding with majority of sputum specimen (38\%) is seen in a study by Ahmad OB et al. ${ }^{7}$ and on contrary certain study like Patel $\mathrm{H}$ et al shows sputum specimen(3\%) to be among the minority specimens and wound or pus specimen $(70 \%)$ to be the majority. Pus specimen is the second most common samplein our study. ${ }^{8}$ Another study by Chanderet al showed pus specimen to be around $27.60 \%$ and sputum sample to be $24.10 \%$ and third common was urine sample with $20.70 \%$. This study also shows high vaginal swab incidence of P.aeruginosa to be around 3.45 which is quite lower than our study. ${ }^{9}$ More than $80 \%$ of sample is obtained from pus and sputum specimen in certain studies in India by Mohanasundaramet $a l,{ }^{10}$ Arora et $a l^{l 1}$ and Chanderet al. $^{9}$

There has been an alarming raise in resistance to different anti-pseudomonal drugs specifically among hospital strains world-wide. ${ }^{12,13}$ This pose a serious problem in the management of disease due to these organisms. The antibiogramof $P$. aeruginosa to the eight anti-microbial agents tested shows a varied pattern among the isolates studied. In this study all the $P$. aeruginosa isolates were found to be sensitive to imipenem and this may be attributed to the limited use of imipenem in this hospital. This is consistent with a report published in 2002 in Mangalore, India. ${ }^{14}$ Certain other recent studies show development of various levels of resistance to imipenem. ${ }^{10,11,15,4}$

Amikacin, Ciprofloxacin and Gentamicin shows $96.8 \%$ sensitivity .These are the most effective drugs for routine use against P.aeruginosa. ${ }^{9}$ A study Nepal ${ }^{16}$ shows amikacin (81.4\% sensitive) and ciprofloxacin (70.3\% sensitive) among $P$. aeruginosa strains examined. A Study by Ahmed et al in 2013 shows sensitivity to Amikacin, Ciprofloxacin and Gentamycin to be $80.5 \%, 43.6 \%$ and $56.1 \%$ respectively. ${ }^{17}$ In 
contrast another recent study shows sensitivity to Amikacin, Ciprofloxacin and Gentamycin to be $18.96 \%, 79.31 \%$ and $13.79 \%$ respectively. ${ }^{18}$

From studies like Hancock et al, ${ }^{19}$ Quinn et al, ${ }^{20}$ Saderet $a l^{21}$ high rate of resistance against carbapenem, quinolones and third generation cephalosporin had been detected in Pseudomonas aeruginosa. Among Cephalosporins highest resistance was observed among $1^{\text {st }}$ generation cefazolin $(83.9 \%)$ followed by $2^{\text {nd }}$ generation Cefotaxime (17.2\%) and Ceftazidime $(23.7 \%)$ the least was seen among $4^{\text {th }}$ generation Cefepime which showed $6.5 \%$ resistance. This was comparable to a study by Ahmed OB et al. ${ }^{7}$ which showed $29.6 \%, 28.7 \%, 32.4 \%$ resistance to Cefotaxime, Ceftazidime and Cefepime respectively. In contrast a study by Fathimaet al shows $40 \%$ resistance among Cefepime $^{22}$ and a study in Egypt showed increasing trends of resistance to cephalosporins like $77.2 \%$, $91.2 \%$ and $98.2 \%$ to Cefotaxime, Ceftazidime and Cefepime respectively. ${ }^{17}$ Another study also revealed increase in trend of resistance pattern by showing 68\% resistance to both Ceftazidime and Cefepime. ${ }^{6}$

In this study Colistin and Aztreonam were not used.They both are used as last resort of antibiotics in treatment of P.aeruginosa. The toxic effects of Colistin should be weighed before therapeutic use.

\section{Conclusion}

In conclusion, restriction of antibiotic usage must be formatted by each institution, to combat the emergence of multi drug resistant $P$. aeruginosa. Imipenem was the only anti-pseudomonal drug against which all isolates of $P$. aeruginosa were fully sensitive. We recommend a restricted and a more rational use of this drug in this hospital setting. Amikacin, ciprofloxacin and semi-synthetic penicillin with betalactamase inhibitors are the preferred drugs for optimal management of infections caused by $P$. aeruginosa. The lack of newer antimicrobial agents with activities against $P$. aeruginosa, makes periodic studies on the antimicrobial resistance patterns very important.A solution can be brought by continuous efforts of an Infection control committee to promote greater understanding of this problem. Adequate hand washing to prevent spread of organism should be encouraged. Better surgical and medical care should be provided to patients during hospital stay.

Funding: No funding sources.

Conflict of interest: None declared.

\section{References}

1. Brooks, G-F, Butel, Carroll, K.C., Morse, S.A (2007), Jawertz, Melnick and Adelberg medical microbiology ( $\left.24^{\text {th }} \mathrm{Ed}\right), \mathrm{P}$ 832. The McGraw-Hill.USA

2. Ryan, K.J (2004). Pseudomonas and other opportunistic gram-negative bacilli. In Sherris medical microbiology (4 $4^{\text {th }}$ ED). Ryan, K. J., Ray, C.G. (Eds) p-385. McGrawHill Companies, USA.
3. Babay, H.A.H. Antimicrobial resistance among clinical isolates of psedudomonas aeruginosa from patients in a teaching hospital, Riyadh, Saudi Arabia, 2001-2005. Jpn J Infect Dis 2007;60:123-5.

4. Javiya VA, Ghatak SB, Patel KR, Patel JA. Antibiotic susceptibility patterns of Pseudomonas aeruginosa at a tertiary care hospital in Gujarat, India. Indian J Pharmacol 2008;40(5):230-34. doi:10.4103/0253-7613.44156.

5. Tamil Selvi Sivanmaliappan and Murugan Sevanan. Antimicrobial Susceptibility Patterns of Pseudomonas aeruginosa from Diabetes Patients with Foot Ulcers. Int $J$ Microbiol .doi:10.1155/2011/605195.

6. Golshani Z, Ahadi AM, Sharifzadeh A. Antimicrobial Susceptibility Pattern of Pseudomonas aeruginosa Isolated from Patients Referring to Hospitals. Arch Hyg Sci 2012;1(2):48-53.

7. Ahmed OB. Incidence and Antibiotic Susceptibility Pattern of Pseudomonas aeruginosa Isolated from Inpatients in Two Tertiary Hospitals. Clin Microbiol 2016;5:248. doi:10.4172/2327-5073.1000248.

8. Patel H, Garala RN. Antibiotic susceptibility pattern of pseudomonas aeruginosa isolated at SSG hospital Baroda. J Res Med Den Sci 2014;2(1):84-7.

9. Chanderanil, Raza mohammadshahid. Antimicrobial susceptibility patterns of pseudomonas aeruginosa clinical isolates at a tertiary care hospital in kathmandu, nepal. Asian J Pharm Clin Res 2013;6(Suppl 3):235-38.

10. Mohanasoundaram KM. The antibiotic resistance pattern in the clinical isolates of Pseudomonas aeruginosa in a tertairy care hospital; 2008-2010 (A 3 year study). J Clin Diagn Res 2011;5(3):491-94.

11. Arora D, Jindal N, Kumar R, Romit. Emerging antibiotic resistance in Pseudomonas aeruginosa. Int J Pharm PharmSci 2011;3(2):82-4.

12. Orrett FA. Antimicrobial susceptibility survey of Pseudomonas aeruginosa strains isolated from clinical sources. J Natl Med Assoc 2004;96(8):1065-69.

13. Chen HY, Yuan M, Livermore DM. Mechanisms of resistance to beta-lactam antibiotics amongst Pseudomonas aeruginosa isolates collected in the United Kingdom in 1993. J Med Microbiol 1995;43:300-9.

14. Shenoy S, Baliga S, Saldanha DR, Prashanth HV. Antibiotic sensitivity patterns of Pseudomonas aeruginosa strains isolated from various clinical specimens. Indian $J$ Med Sci 2002;56(9):427-30.

15. Al-KabsiAM, Yusof MYBM, Sekaran SD. Antimicrobial resistance pattern of clinical isolates of Pseudomonas aeruginosa in the University of Malaya Medical Center, Malaysia. Afr J Microbiol Res 2011;5(29):5266-72.

16. Koirala P, Bhatta DR, Ghimire P, Pokhrel BM, Devkota U. Bacteriological profile of tracheal aspirates of the patients attending a neuro-hospital of Nepal. Int J Life Sci 2010;4:60-65.

17. Ahmed Bakr Mahmoud, Wafaa Ahmed Zahran, Ghada Rashad Hindawi, AzaZaghlolLabib and RashaGalal. Prevalence of Multidrug-Resistant Pseudomonas aeruginosa in Patients with Nosocomial Infections at a University Hospital in Egypt, with Special Reference to Typing Methods. J Virol Microbiol 10.5171/2013.290047.

18. Indu Biswal, Balvinder Singh Arora, Dimple Kasana, Neetushree4.Incidence of Multidrug Resistant Pseudomonas Aeruginosa Isolated from Burn Patients and Environment of Teaching Institution.DOI: 10.7860/JCDR/2014/7483.4383.

19. Hancock REW. Resistance mechanism in pseudomonas aeruginosa and other nonfermentative gram-negative bacteria. Clin Infect Dis 1998;27:289-99. 
20. Quinn JP. Clinical problems posed by multiresistantnonfermenting gram negative pathogens. Clin Infect Dis 1998;27:117-24.

21. Sader HS, Jones RN, Gales AC et al (1998)

Antimicrobial susceptibility patterns for pathogens isolated from patients in Latin American medical centers with a diagnosis of pneumonia: analysis of results from the SENTRY antimicrobial surveillance program (1997). SENTRY Latin American study group. DiagnMicrobiol Infect Dis 32:289-301.
22. Fatima. Antimicrobial susceptibility pattern of clinical isolates of Pseudomonas aeruginosa isolated from patients of lower respiratory tract infections. SpringerPlus 2012 1:70.

How to cite this article: Kaliyamoorthi D., K. Sharanya, Saikumar C., G. Sridhar, Boopathy. Antimicrobial resistance pattern of Pseudomonas aeruginosa in a tertiary level hospital in Southern India. Int J Med Microbiol Trop Dis 2018;4(4):261-65. 